\title{
Static spin-dependent forces between heavy quarks in the classical approximation to dual QCD
}

\author{
M. Baker \\ University of Washington, Seattle, Washington 98105 \\ James S. Ball \\ University of Utah, Salt Lake City, Utah 84112 \\ F. Zachariasen \\ California Institute of Technology, Pasadena, California 91125
}

(Received 15 August 1991)

\begin{abstract}
We compute the static spin-dependent forces $V_{S}(R)$ (proportional to $\sigma_{1} \cdot \sigma_{2}$ ) and $V_{T}(R)$ (proportional to $3 \sigma_{1} \cdot \hat{\mathbf{R}} \sigma_{2} \cdot \hat{\mathbf{R}}-\sigma_{1} \cdot \sigma_{2}$ ) between two quarks separated by $\mathbf{R}$. This is done by treating the (weak) spin-dependent effects as a perturbation on the spin-independent potentials and fields computed earlier for dual QCD. What results is a definite prediction for the heavy-quark potentials which are similar to, but different in form from, those used in phenomenological treatments. Calculations of the masses and splittings of heavy-quark states using our potentials will provide a further test of the dual superconductor picture of QCD.
\end{abstract}

\section{INTRODUCTION}

We have recently reported [1] a classical calculation of the static potential between heavy quarks in dual QCD, which is a close analogue of the calculation of the force between two magnetic monopoles in a Landau-Ginzburg superconductor [2]. In this paper we wish to extend our calculation to include the spin-dependent forces between static quarks.

In many ways this is an easier calculation than that of the potential. A spin produces a magnetic dipole, which can be represented as a closely spaced pair of oppositely charged magnetic monopoles. Thus the spin-dependent interaction is the same as the interaction of four magnetic monopoles, arranged as two magnetic dipoles.

Inserting (color-) magnetic monopole sources in the field equations of dual QCD is easy; they simply appear as sources in the dual of Gauss's law:

$$
\mathcal{D}_{C} \cdot \mathbf{H}+i g\left[\mathcal{D}_{0} \mathbf{B} \cdot \mathbf{B}\right]=\sum_{i} m_{i} \delta^{3}\left(\mathbf{x}-\mathbf{x}_{i}\right) \equiv \rho_{M},
$$

where the right-hand side represents a set of monopoles with magnetic charge $m_{i}$ located at $\mathbf{x}_{i}$, and on the lefthand side $\mathbf{H}$ is the color-magnetic field and $\mathcal{D}_{C}$ and $\mathcal{D}_{0}$ are the dual covariant derivatives. Thus, in terms of the dual potential $C_{\mu}$, we have

$$
\begin{aligned}
& \mathbf{H}=-\partial_{0} \mathbf{C}-\nabla C_{0}-i g\left[\mathbf{C}, C_{0}\right], \\
& \mathcal{D}_{0}=\partial_{0}-i g\left[C_{0},\right]
\end{aligned}
$$

and

$$
\mathcal{D}_{C}=\boldsymbol{\nabla}+i g[\mathbf{C} \cdot,]
$$

In the absence of quark sources we found a classical fluxtube solution carrying a single unit of $Z_{3}$ flux. The dual potential $\mathbf{C}$ was in the direction $Y \equiv \lambda_{8} / \sqrt{3}$. In order to absorb this flux, quark sources $\rho$, when present, must also be in the $Y$ direction in color space, as was the case in I. Likewise the potential $C_{0}$ and the magnetic sources $\rho_{M}$ must be in the $Y$ direction.

The remainder of the dual QCD field equations are unaffected by the presence of the monopoles [3]. We are thus led to a rather simple set of coupled nonlinear differential equations for $C_{0}, \mathbf{C}$ and the color-magnetic induction $\mathbf{B}$, which in the case of cylindrical symmetry can be solved quite easily on a computer. Unfortunately, to compute the two independent spin-spin potentials, it is necessary to have solutions for dipoles oriented both parallel and perpendicular to the $z$ axis which are no longer cylindrically symmetric.

For this reason we shall calculate $C_{0}$, and hence $\mathbf{H}$, in perturbation theory, starting from the cylindrically symmetric solutions obtained in the calculation of the static potential [1]; that is, in the differential equation for $C_{0}$ we shall use the values of $\mathbf{C}$ and $\mathbf{B}$ taken from the static potential problem, rather than attempt to solve the entire coupled system of nonlinear equations in three dimensions. This neglects the feedback of the spindependent effects on the fields associated with the static spin-independent potential, and should be an excellent approximation, given the fact that the spin-dependent forces are much weaker than the spin-independent force.

\section{FIELD EQUATIONS}

First of all it will be convenient to define $C_{M}(\mathbf{x})$ as the scalar potential which would be produced by a given set of fixed magnetic monopoles in the perturbative vacuum, 
i.e., in free space. So we define

$$
\nabla^{2} C_{M}(\mathbf{x})=-\sum_{i} m_{i} \delta^{3}\left(\mathbf{x}-\mathbf{x}_{i}\right)
$$

whereupon, for four monopoles arranged in two dipoles, we find

$$
C_{M}(\mathbf{x})=\frac{1}{4 \pi}\left(\frac{\mathbf{d}_{1} \cdot\left(\mathbf{x}-\mathbf{x}_{1}\right)}{\left|x-x_{1}\right|^{3}}+\frac{\mathbf{d}_{2} \cdot\left(\mathbf{x}-\mathbf{x}_{2}\right)}{\left|x-x_{2}\right|^{3}}\right),
$$

where $\mathbf{d}_{1}$ and $\mathbf{d}_{2}$ are the two dipole moments. We also define $\mathbf{R}=\mathbf{x}_{1}-\mathbf{x}_{2}$ as their separation.

Next we define the full (dual) scalar potential $C_{0}$ as

$$
C_{0}=\left(c_{0}+C_{M}\right) Y \text {. }
$$

Then, using the same ansatz as in I for $\mathbf{C}$ and $\mathbf{B}$, namely, in cylindrical coordinates with the quarks lying on the $z$ axis,

$$
\begin{aligned}
& \mathbf{C}=C(\mathbf{x}) \hat{\mathbf{e}}_{\varphi} Y, \\
& \mathbf{B}=B(\mathbf{x}) \hat{\mathbf{e}}_{x}\left(\lambda_{7}\right)+B(\mathbf{x}) \hat{\mathbf{e}}_{y}\left(-\lambda_{5}\right)+B_{3}(\mathbf{x}) \hat{\mathbf{e}}_{z}\left(\lambda_{2}\right),
\end{aligned}
$$

the field equations are readily written down. They are (using the same scalings as in I)

$$
\nabla^{\prime 2} c_{0}^{\prime}-\frac{3}{2} g^{\prime 2} B^{2}\left(c_{0}^{\prime}+C_{M}^{\prime}\right)=0
$$

$$
\begin{aligned}
& \tilde{\nabla}^{\prime 2} c^{\prime}-\frac{3}{2} g^{2} B^{\prime 2}\left(c^{\prime}+C_{D}^{\prime}\right)=0 \\
& \nabla^{\prime 2} B^{\prime}-g^{\prime 2}\left[\left(c^{\prime}+C_{D}^{\prime}\right)^{2}-\left(c_{0}^{\prime}+C_{M}^{\prime}\right)^{2}\right] B^{\prime}=\frac{1}{2} \frac{\partial W^{\prime}}{\partial B^{\prime}}
\end{aligned}
$$

and

$$
\nabla^{2} B_{3}^{\prime 2}=\frac{\partial W^{\prime}}{\partial B_{3}^{\prime}}
$$

These will be recognized as exactly the equations for the static spin-independent potential given in I (we recall that $C_{D}$, defined in $\mathrm{I}$, is the Dirac string potential associated with the two quarks) supplemented by the dual Gauss law [Eq. (2.6a)] and including the effect of $C_{0}$ on B via Eq. (2.6c).

Since we shall calculate perturbatively in the spindependent potential, we will drop the $c_{0}^{\prime}+C_{M}^{\prime}$ term from $(2.6 \mathrm{c})$. Then $(2.6 \mathrm{~b})$ through $(2.6 \mathrm{~d})$ are exactly the equations solved in I, so that $c^{\prime}, B^{\prime}$, and $B_{3}^{\prime}$ may be taken as known, and used as input in Eq. (2.6a). (They are functions only of $\rho^{\prime}$ and $z^{\prime}$.) This, then, is the only equation which needs to be solved.

To do this, it is convenient to write out, in cylindrical coordinates, the expression for $\mathbf{C}_{M}^{\prime}$ more explicitly. Choosing the dipoles to lie on the $z$ axis at $\pm R / 2$, writing the dipole moment vectors in terms of their components parallel and perpendicular to the $z$ axis, and choosing $\mathbf{d}_{1 \perp}$ in the $\varphi=0$ plane, we have

$$
\begin{aligned}
C_{M}^{\prime} & =\frac{1}{4 \pi}\left(\frac{d_{1 z}(z-R / 2)}{\left[\rho^{2}+(z-R / 2)^{2}\right]^{3 / 2}}+\frac{d_{2 z}(z+R / 2)}{\left[\rho^{2}+(z+R / 2)^{2}\right]^{3 / 2}}\right)+\frac{\rho}{4 \pi} \frac{d_{1 \perp} \cos \varphi}{\left[\rho^{2}+(z-R / 2)^{2}\right]^{3 / 2}}+\frac{\rho}{4 \pi} \frac{d_{2 \perp} \cos \left(\varphi+\varphi_{0}\right)}{\left[\rho^{2}+(z+R / 2)^{2}\right]^{3 / 2}} \\
& \equiv d_{1 z} C_{M z}^{1}+d_{2 z} C_{M z}^{2}+d_{1 \perp} C_{M \perp}^{1} \cos \varphi+d_{2 \perp} C_{M \perp}^{2} \cos \left(\varphi+\varphi_{0}\right)
\end{aligned}
$$

The equation for $c_{0}^{\prime}$ is linear, so the analogous breakup for this function is also natural:

$$
\begin{aligned}
c_{0} \equiv & c_{0 z}^{1} d_{1 z}+c_{0 z}^{2} d_{2 z} \\
& +c_{0 \perp}^{1} d_{1 \perp} \cos \varphi+c_{0 \perp}^{2} d_{2 \perp} \cos \left(\varphi+\varphi_{0}\right) .
\end{aligned}
$$

Next we note the identity

$$
\nabla^{2} \cos \left(\varphi+\varphi_{0}\right) f(\rho, z)=\cos \left(\varphi+\varphi_{0}\right) \tilde{\nabla}^{2} f(\rho, z)
$$

where

$$
\nabla^{2}=\frac{1}{\rho} \frac{\partial}{\partial \rho} \rho \frac{\partial}{\partial \rho}+\frac{\partial^{2}}{\partial z^{2}}
$$

and

$$
\tilde{\nabla}^{2}=\nabla^{2}-1 / \rho^{2} .
$$

Consequently Eq. (2.6a) boils down to the four equations

$$
\begin{aligned}
& \nabla^{2} c_{0 z}^{\prime 1,2}-\frac{3}{2} g^{2} B^{2}\left(c_{0 z}^{\prime 1,2}+C_{M z}^{\prime 1,2}\right)=0, \\
& \tilde{\nabla}^{2} c_{0 \perp}^{\prime 1,2}-\frac{3}{2} g^{2} B^{\prime 2}\left(c_{0 \perp}^{\prime 1,2}+C_{M \perp}^{1,2}\right)=0 .
\end{aligned}
$$

\section{SOLUTIONS}

In our previous calculation the behavior of $C_{D}^{\prime}$ and the location of the string determined the boundary conditions for $B$ along the $z$ axis. These boundary conditions eliminated all numerical problems related to the source singularities in $(2.6 \mathrm{c})$. In our present calculation the dipole potential $C_{M}^{\prime}$ is much more singular and the detailed behavior of the fields near the source must be analyzed to determine the correct numerical procedures.

We will first determine the behavior of $B$ near the point source. In spherical coordinates, the Dirac field is

$$
C_{D}^{\prime}=\frac{1}{2 g^{\prime} r} \frac{1+\cos \theta}{\sin \theta} \text {. }
$$

Here we have chosen the string direction along the negative $z$ axis. Since $c^{\prime}$ vanishes along the $z$ axis, near the source, Eq. (2.6c) has the form

$$
\nabla^{\prime 2} B^{\prime}=\frac{B^{\prime}}{4 r^{2}} \frac{(1+\cos \theta)^{2}}{\sin ^{2} \theta} .
$$

This equation is separable and the form $B^{\prime}=r^{\alpha} T(x)$ 
where $x=\cos \theta$ leads to a second-order differential equation for $T(x)$ which has three regular singular points. The solution which is finite at $x=1$ is

$$
T(x)=\sqrt{1-x}{ }_{2} F_{1}\left(1-\beta, 1+\beta ; 2 ; \frac{1-x}{2}\right),
$$

where $\beta=\sqrt{\alpha(\alpha+1)+\frac{1}{2}}$ and ${ }_{2} F_{1}$ is the usual hypergecmetric function. The requirement that $B^{\prime}$ be finite at $x=-1$ forces $\beta$ to be an integer. The smallest value of $\alpha$ for which this occurs is $\alpha=(\sqrt{3}-1) / 2$. The resulting solution for $B^{\prime}$ is

$$
B^{\prime}=b \sqrt{1-\cos \theta} r^{(\sqrt{3}-1) / 2} .
$$

Here $b$ is an arbitrary constant as far as our analysis is concerned, although it is of course determined by the global solution for $B^{\prime}$. Our numerical solution for $B^{\prime}$ is in excellent agreement with this form near the sources.

Given the form of $B^{\prime}$ near a source, it is now a simple matter to determine the behavior of $c_{0 \perp}^{\prime}$ and $c_{0 z}^{\prime}$. Choosing the dipole to be located at the origin, and keeping only the most singular terms ( $c_{0}^{\prime}$ is much less singular than $C_{M}^{\prime}$ ), Eqs. (2.12) become

$$
\nabla^{2} c_{0 z}^{\prime}=\frac{3}{2} g^{2} B^{\prime 2} C_{M z}^{\prime}=\kappa d_{z} \cos \theta(1-\cos \theta) r^{\sqrt{3}-3}
$$

and

$$
\tilde{\nabla}^{2} c_{0 \perp}^{\prime}=\frac{3}{2} g^{2} B^{\prime 2} C_{M \perp}^{\prime}=\kappa d_{\perp} \sin \theta(1-\cos \theta) r^{\sqrt{3}-3} .
$$

Here the constant $\kappa=(3 / 8 \pi) g^{\prime 2} b^{2}$. The $r$ dependence of $c_{0 z}$ and $c_{0 \perp}$ must therefore be $r^{\sqrt{3}-1}$. The field components then have the form

$$
c_{0 z}=d_{z} T_{z}(\cos \theta) r^{\sqrt{3}-1}
$$

and

$$
c_{0 \perp}=d_{\perp} \sin \theta T_{\perp}(\cos \theta) r^{\sqrt{3}-1} .
$$

The $T$ 's are the solutions to the inhomogeneous differential equations

$$
\frac{d}{d x}\left(1-x^{2}\right) \frac{d}{d x} T_{z}(x)+(3-\sqrt{3}) T_{z}(x)=\kappa x(1-x)
$$

and

$$
2 \frac{d}{d x}\left(1-x^{2}\right) \frac{d}{d x} T_{\perp}(x)+(1-\sqrt{3}) T_{\perp}(x)=\kappa(1-x)
$$

which are the polynomials

$$
T_{z}(x)=\kappa\left(-\frac{1}{3}+\frac{x}{1-\sqrt{3}}+\frac{x^{2}}{3+\sqrt{3}}\right)
$$

and

$$
T_{\perp}(x)=\kappa\left(\frac{1}{1-\sqrt{3}}+\frac{x}{3+\sqrt{3}}\right) \text {. }
$$

The general fields near the dipole source are then those given in (3.6) plus the regular solutions to the homogeneous equations. Note also that the solution due to one source in the neighborhood of the other source is only the solution to the homogeneous equation as $B^{\prime}$ vanishes and the other terms on the right-hand side of (3.5) are nonsingular.

From our analysis we see that $c_{0 \perp}^{\prime}$ vanishes on the $z$ axis and will require no special treatment. On the other hand, $c_{0 z}^{\prime}$ goes to a nonzero constant. In our usual numerical procedure, the value at the dipole position would be proportional to the infinite quantity $B^{\prime 2} C_{M z}^{\prime}$. To evaluate $c_{0 z}^{\prime}$ at this point, we use the form given by (3.6a) to interpolate the value from the three adjacent grid points. The resulting numerical solutions near the dipole sources are in very good agreement with our analytic forms.

In our previous calculations described in I, we used the fact that the fields were symmetric under $z$ goes to $-z$ to reduce the volume considered to the half-space $z>0$. As a result, only half the number of mesh points were required to produce a given step size (density of mesh points in the $\rho-z$ plane). The same procedure can be used in this calculation if we change from fields labeled 1 or 2 according to the source position to linear combinations that are even or odd in $z$ as follows:

$$
\begin{aligned}
& C_{M z}^{\prime e}=C_{M z}^{\prime 1}-C_{M z}^{\prime 2} \\
& C_{M z}^{\prime 0}=C_{M z}^{\prime 1}+C_{M z}^{\prime 2} \\
& C_{M \perp}^{\prime e}=C_{M \perp}^{\prime 1}+C_{M \perp}^{\prime 2} \\
& C_{M \perp}^{\prime 0}=C_{M \perp}^{\prime 1}-C_{M \perp}^{\prime 2}
\end{aligned}
$$

and

$$
\begin{aligned}
& c_{0 z}^{\prime e}=c_{0 z}^{\prime 1}-c_{0 z}^{\prime 2}, \\
& c_{0 z}^{\prime 0}=c_{0 z}^{\prime 1}+c_{0 z}^{\prime 2}, \\
& c_{0 \perp}^{\prime e}=c_{0 \perp}^{\prime 1}+c_{0 \perp}^{\prime 2}, \\
& c_{0 \perp}^{\prime 0}=c_{0 \perp}^{\prime 1}-c_{0 \perp}^{\prime 2} .
\end{aligned}
$$

The form of the field equations (2.12) is unchanged:

$$
\nabla^{2} c_{0 z}^{\prime e, 0}-\frac{3}{2} g^{\prime 2} B^{\prime 2}\left(c_{0 z}^{\prime e, 0}+C_{M z}^{\prime e, 0}\right)=0,
$$

and

$$
\tilde{\nabla}^{2} c_{0 \perp}^{\prime e, 0}-\frac{3}{2} g^{\prime 2} B^{\prime 2}\left(c_{0 \perp}^{\prime e, 0}+C_{M \perp}^{e, 0}\right)=0 .
$$

These four equations need only be solved for $z>0$ subject to the boundary conditions on the $z=0$ plane that the odd functions vanish and $d c^{\prime} / d z=0$ for the even functions.

We used the same general numerical techniques described in I to solve Eqs. (3.11) for a range of values of 
$R$. Because $B^{\prime}$ values from our previous calculation were a necessary input for this calculation, we chose not to vary the lattice size but to use the largest one on which the solution for $B^{\prime}$ was obtained. The typical lattice size was $64 \times 64$. For each value of $R$ we used the GaussSeidel method with the successive overrelaxation (SOR) technique to produce the solutions to (3.11). The rate of convergence required 400 to 600 iterations to produce accurate results.

\section{THE ENERGY}

In our scaled units, the dual QCD Lagrangian, including only the fields $c_{0}, c, B$, and $B_{3}$, is given by [1]

$$
\begin{gathered}
\mathcal{L}=\lambda\left(\left(-\tilde{F}_{0}\right)\right)^{2}\left\{\frac{2}{3}\left(c^{\prime}+C_{D}^{\prime}\right) \tilde{\nabla}^{2}\left(c^{\prime}+C_{D}^{\prime}\right)+B^{\prime} \nabla^{\prime 2} B^{\prime}+\frac{1}{2} B_{3}^{\prime} \nabla^{\prime 2} B_{3}^{\prime}-\frac{2}{3}\left(c_{0}^{\prime}+C_{M}^{\prime}\right) \nabla^{2}\left(c_{0}^{\prime}+C_{M}^{\prime}\right)-\frac{4}{3}\left(c_{0}^{\prime}+C_{M}^{\prime}\right) \rho_{M}\right. \\
\left.-g^{\prime 2} B^{\prime 2}\left[\left(c^{\prime}+C_{D}^{\prime}\right)^{2}-\left(c_{o}^{\prime}+C_{M}^{\prime}\right)^{2}\right]-W^{\prime}\right\}
\end{gathered}
$$

where $\rho_{M}$ is the magnetic charge density, Eq. (1.1). As is the case for time-independent solutions, the Hamiltonian is the negative of the Lagrangian. Using Eq. (2.1) to eliminate the magnetic charge density and the field equation (2.6a), we find that the scaled spin-dependent part of the Hamiltonian is

$$
\mathcal{H}_{\text {spin }}^{\prime}=-\left[\frac{2}{3}\left(c_{0}^{\prime}+C_{M}^{\prime}\right) \nabla^{\prime 2} C_{M}^{\prime}\right] .
$$

The fact that $c_{0}^{\prime 1,2}+C_{M}^{1,2}$ vanishes exponentially as a function of distance from its source means that the complete spin potential will vanish exponentially at large $R$. We note that, from (2.1), for four monopoles arranged in two dipoles,

$$
\nabla^{\prime 2} C_{M}^{\prime}(\mathbf{x})=\mathbf{d}_{1} \cdot \nabla^{\prime} \delta^{3}\left(\mathbf{x}-\mathbf{x}_{1}\right)+\mathbf{d}_{2} \cdot \nabla^{\prime} \delta^{3}\left(\mathbf{x}-\mathbf{x}_{2}\right)
$$

and hence

$$
\int d^{3} \mathbf{x}^{\prime} C_{M}^{\prime} \nabla^{\prime 2} C_{M}^{\prime}=-\mathbf{d}_{1} \cdot \nabla^{\prime} C_{M}^{\prime}\left(\mathbf{x}_{1}\right)-\mathbf{d}_{2} \cdot \nabla^{\prime} C_{M}^{\prime}\left(\mathbf{x}_{2}\right)
$$

This contributes the usual free space dipole-dipole interaction to the energy:

$$
V_{\text {spin }}^{\text {free space }}=-\frac{4}{3} \frac{1}{4 \pi} \frac{3 \mathbf{d}_{1} \cdot \hat{\mathbf{R}} \mathbf{d}_{2} \cdot \hat{\mathbf{R}}-\mathbf{d}_{1} \cdot \mathbf{d}_{2}}{R^{3}} .
$$

The interaction energy from the cross term between $c_{0}^{\prime}$ and $C_{M}^{\prime}$ can also be evaluated using (4.3),

$$
\int d^{3} \mathbf{x}^{\prime} c_{0}^{\prime} \nabla^{\prime 2} C_{M}^{\prime}=-\mathbf{d}_{1} \cdot \nabla^{\prime} c_{0}^{\prime 2}\left(\mathbf{x}_{1}\right)-\mathbf{d}_{2} \cdot \nabla^{\prime} c_{0}^{\prime 1}\left(\mathbf{x}_{2}\right)
$$

and, as we have seen in Sec. III, these terms are finite.

The complete $V_{\text {spin }}$ is then

$$
V_{\mathrm{spin}}=V_{z} d_{1 z} d_{2 z}+V_{\perp} \mathbf{d}_{1 \perp} \cdot \mathbf{d}_{2 \perp}
$$

where

$$
V_{z}=-\frac{2}{3 \pi} \frac{1}{R^{3}}+\frac{4}{3} \frac{\partial c_{0 z}^{\prime 2}\left(\mathbf{x}_{1}\right)}{\partial z}
$$

and

$$
V_{\perp}=\frac{1}{3 \pi} \frac{1}{R^{3}}+\frac{4}{3} \frac{\partial c_{0 \perp}^{\prime 2}\left(\mathbf{x}_{1}\right)}{\partial \rho} .
$$

A comparison of this form of $V_{\text {spin }}$ with the standard notation

$V_{\text {spin }}=V_{S}(R) \mathbf{d}_{1} \cdot \mathbf{d}_{2}+V_{T}(R)\left(3 \mathbf{d}_{1} \cdot \hat{\mathbf{R}} \mathbf{d}_{2} \cdot \hat{\mathbf{R}}-\mathbf{d}_{1} \cdot \mathbf{d}_{2}\right)$

yields the relations

$$
V_{T}=\frac{1}{3}\left(V_{z}-V_{\perp}\right)
$$

and

$$
V_{S}=\frac{1}{3}\left(V_{z}+2 V_{\perp}\right) .
$$

\section{RESULTS AND COMPARISON WITH EXPERIMENT}

We first replace the dipole moments $\mathbf{d}_{1}$ and $\mathbf{d}_{2}$ in (4.9) by $\left(e / 2 m_{q}\right) \sigma_{1}$ and $-\left(e / 2 m_{q}\right) \sigma_{2}$, corresponding to the intrinsic spin of the quark-antiquark pair.

In Figs. 1 and 2 we show our results for $V_{T}$ and $V_{S}$ which are calculated at the points marked with $\mathrm{a}+$. The

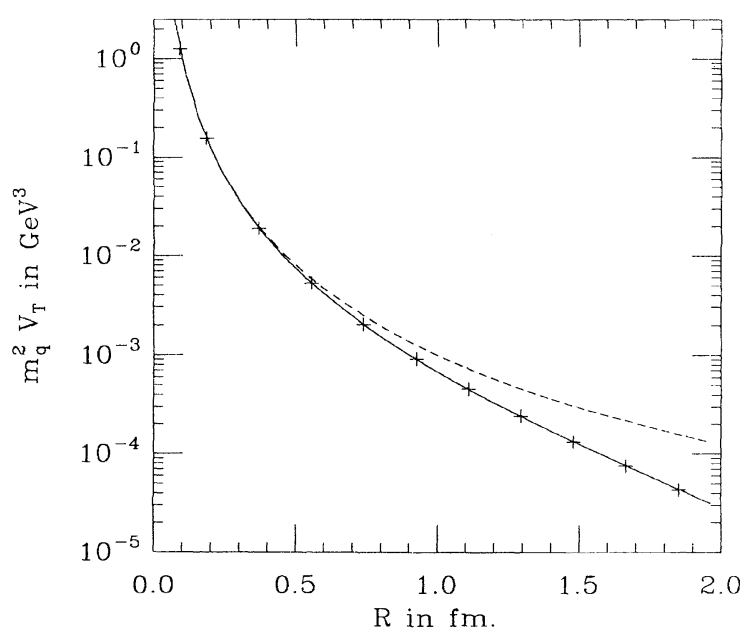

FIG. 1. The spin-dependent potential $m_{q}^{2} V_{T}(R)$ plotted as a function of quark separation $R$ for $\alpha_{s}=0.39$. The +'s are our calculated points, the solid curve is our empirical form and the dashed curve is the free space dipole-dipole interaction. 


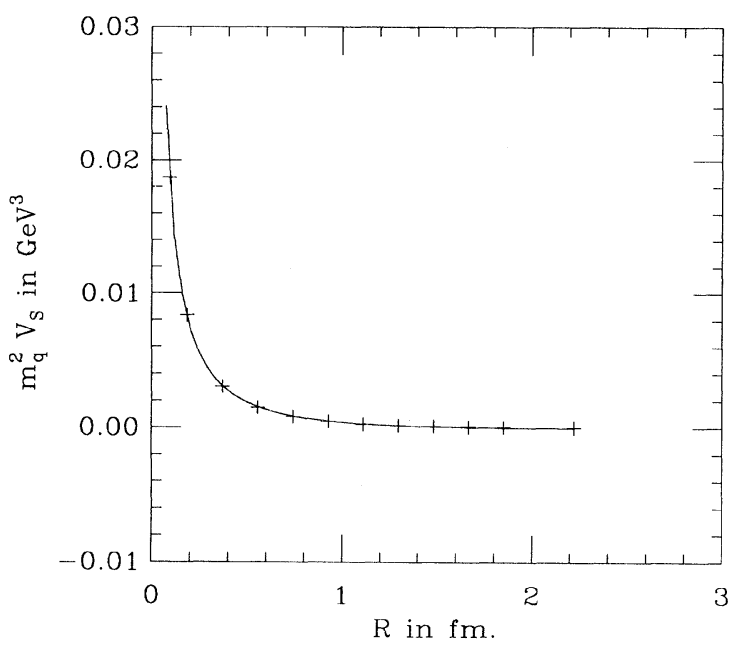

FIG. 2. The spin-dependent potential $m_{q}^{2} V_{S}(R)$ plotted as a function of quark separation $R$ for $\alpha_{s}=0.39$. The +'s are our calculated points and the solid curve is our empirical form.

smooth curves are empirical fits used to interpolate between the points. Because of the $1 / R^{3}$ behavior of $V_{T}$ for small $R$ it was necessary to use a logarthimic scale for $V_{T}$ in order to display its behavior at larger values of $R$. For comparison, the free space dipole-dipole interaction has also been plotted in Fig. 1. To completely specify our results for the heavy-quark potential, we show our results for the spin-independent potential $V$ from $I$ in Fig. 3. Again the +'s are the calculated points and the solid curve is an exponential times the Coulomb term plus the string tension term plus a constant. All of these quantities have been calculated for $g^{\prime 2}=5, \lambda=1.61$, and $\sqrt{-\tilde{F}_{0}^{2}}=420 \mathrm{MeV}$. This choice fixes $\alpha_{s}=\pi / \lambda g^{2}=0.39$.

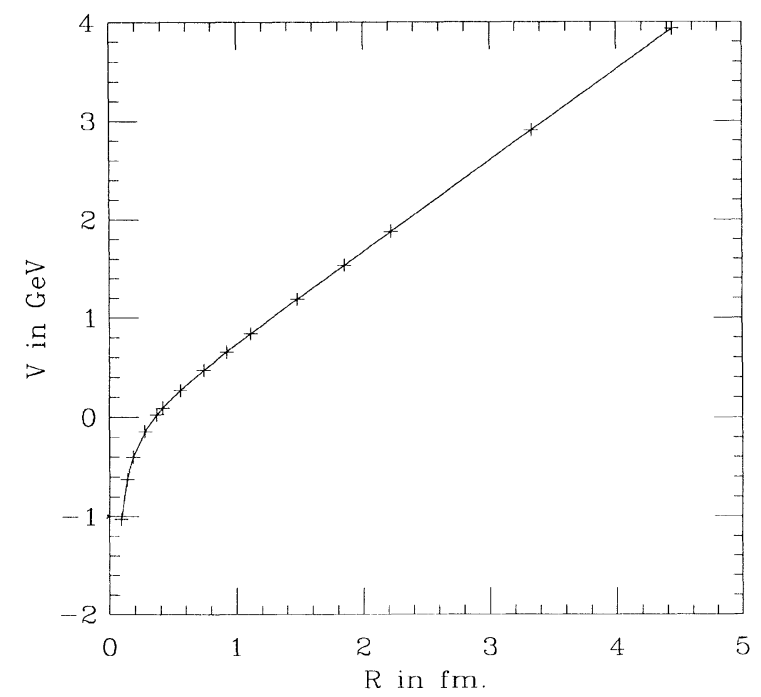

FIG. 3. Fit to the static potential $V(R)$ for $\alpha_{s}=0.39$. The +'s are our calculated points and the solid curve is our empirical form.
The quantities $V_{S}$ and $V_{T}$ are proportional to $\alpha_{s}$ and this fact is made explicit in our empirical formulas.

The reader should be reminded that all of the potential terms which we have calculated depend on $B$ which in turn depends nonlinearly on $g^{\prime 2}$. The other parameters have been adjusted to give good values for $\alpha_{s}$, which, for fixed $g^{\prime 2}$, is only a function of $\lambda$, and the string tension, which is only a function of $\tilde{F}_{0}^{2}$.

We find that $V_{T}$ comes out to be the free space dipoledipole interaction for small $R$ and then falls exponentially for larger values due to the presence of the dual superconducting vacuum. Our empirical form is

$$
\begin{aligned}
V_{T}= & \frac{\alpha_{s} \pi}{m_{q}^{2}}\left(-\lambda \tilde{F}_{0}^{2}\right)^{3 / 2}\left(0.1018+0.0534 x-0.00761 x^{2}\right) \\
& \times \frac{e^{-0.360 x}}{x^{3}}
\end{aligned}
$$

where $x=\sqrt{-\lambda \tilde{F}_{0}^{2}} R$ is the scaled source separation. From our analytic expression for the spin-spin interaction energy, the $R \rightarrow 0$ singularity should be that due to free dipoles. This would require the coefficient of $1 / x^{3}$ in Eq. (5.1) to be $1 / 3 \pi$ which is very close to our fitted value of 0.1018 .

The free space $V_{S}$ has only a $\delta$-function term, so that the entire potential shown in Fig. 2 is a new feature due to the presence of the dual superconducting vacuum. It behaves like $1 / R$ for small $R$ and falls exponentially with a scale similar to that for $V_{T}$.

Up to this point we have only considered fields outside of the region of the source since these are easily treated with our dual potentials. On the other hand, as in ordinary electricity, the behavior of the fields inside the source depends on the nature of the source. For example, in ordinary (nondual) electricity, we have

$$
\boldsymbol{\nabla} \times \mathbf{A}_{\text {dipole }}=\frac{3 \hat{\mathbf{R}}(\mathbf{d} \cdot \hat{\mathbf{R}})-\mathbf{d}}{4 \pi R^{3}}+\frac{2}{3} \mathbf{d} \delta^{3}(\mathbf{R}) .
$$

If we calculate the divergence of the first term on the right-hand side of $\mathrm{Eq}$. (5.2) we obtain

$$
\boldsymbol{\nabla} \cdot \frac{3 \hat{\mathbf{R}}(\mathbf{d} \cdot \hat{\mathbf{R}})-\mathbf{d}}{4 \pi R^{3}}=-\frac{2}{3} \mathbf{d} \cdot \nabla \delta^{3}(\mathbf{R})
$$

which, using Eq. (5.2), is consistent with the requirement that the divergence of the curl vanish. On the other hand, in (Abelian) dual QCD, we have

$$
-\nabla C_{0 \text { dipole }}=\frac{3 \hat{R}(\mathbf{d} \cdot \hat{R})-\mathbf{d}}{4 \pi R^{3}}-\frac{1}{3} \mathbf{d} \delta^{3}(\mathbf{R}) .
$$

The divergence of this expression is in agreement with Eq. (4.3) for a single source. Thus we see that the $\delta$ function part of $\mathbf{H}$ depends on the microscopic source of the quark color-magnetic dipole moment with (5.4) being the limit of two monopoles and (5.2) being the result of a color-electric current.

Since the quark dipole moment is the result of circulating color-electric currents, their treatment in QCD requires some care. In I we were forced to introduce a "string field" to modify the relation between $\mathbf{D}$ and $\mathbf{C}$ 
to treat a color charge source. In this case we modify Eq. (1.2) by adding an $\mathbf{H}_{s}$ to give $\mathbf{H}$ the proper curl [4]:

$$
\mathbf{H}=-\nabla C_{0}+\mathbf{H}_{s}
$$

and

$$
\boldsymbol{\nabla} \times \mathbf{H}=\boldsymbol{\nabla} \times \mathbf{H}_{s}=\boldsymbol{\nabla} \times \mathbf{M},
$$

where $\mathbf{M}$ is the magnetization density given by the dipoles. Thus $\mathbf{H}_{s}$ only exists at the sources:

$$
\mathbf{H}_{s}=\mathbf{M}=\mathbf{d}_{1} \delta^{3}\left(\mathbf{x}-\mathbf{x}_{1}\right)+\mathbf{d}_{2} \delta^{3}\left(\mathbf{x}-\mathbf{x}_{2}\right) .
$$

Let us return to the Lagrangian and our field equations to see what has been changed by introducing $\mathbf{H}_{s}$. The term

$$
-\frac{2}{3}\left(c_{0}^{\prime}+C_{M}^{\prime}\right) \nabla^{2}\left(c_{0}^{\prime}+C_{M}^{\prime}\right)
$$

is $\frac{2}{3} H^{2}$ which should be replaced with $\mathbf{H}$ as given in Eq. (5.5). Because we no longer have any magnetic charge, $\rho_{M}$ is zero and there is no source term in the Lagrangian. The new cross term between $\boldsymbol{\nabla} C_{0}$ and $\mathbf{H}_{s}$ is, after integration by parts, exactly equal to the old source term. As a result the only new term in the Lagrangian is $H_{s}^{2}$ which has no effect on the field equations but contributes to the interaction energy. Its only effect is to change the $\delta$-function term in $V_{S}$ to that produced by dipoles resulting from color-electric currents.

Our empirical form for $V_{S}$, including the $\delta$ function, is then

$$
\begin{aligned}
V_{S}=\frac{8 \pi \alpha_{s}}{9 m_{q}^{2}} \delta^{3}(\mathbf{R})+ & \frac{\alpha_{s} \pi}{m_{q}^{2}}\left(-\lambda \tilde{F}_{0}^{2}\right)^{3 / 2}(0.0311-0.00361 x) \\
& \times \frac{e^{-0.530 x}}{x} .
\end{aligned}
$$

Finally, the simple empirical fit to the spin- independent potential between heavy quarks numerically computed in $\mathrm{I}$ is

$$
\begin{aligned}
V(R)= & -\frac{4}{3} \frac{\alpha_{s}}{R} e^{-0.643 x}+1.0324 R\left(-\tilde{F}_{0}^{2}\right) \\
& -0.5164\left(-\frac{\tilde{F}_{0}^{2}}{\lambda}\right)^{1 / 2} .
\end{aligned}
$$

Thus altogether the static potential predicted by dual QCD in the classical approximation is

$V(R)+V_{T}(R)\left(3 \boldsymbol{\sigma}_{1} \cdot \hat{\mathbf{R}} \boldsymbol{\sigma}_{2} \cdot \hat{\mathbf{R}}-\boldsymbol{\sigma}_{1} \cdot \boldsymbol{\sigma}_{2}\right)+V_{S}(R) \boldsymbol{\sigma}_{1} \cdot \boldsymbol{\sigma}_{2}$,

with $V(R)$ given by $(5.9), V_{S}(R)$ by $(5.8)$ and $V_{T}(R)$ given by (5.1).

To compare our predicted static spin-dependent potentials to phenomenologically determined potentials is very difficult. There is a vast array of phenomenological potentials on the market [5], most of which differ greatly, a few of which have some distant motivation in QCD, and many of which are purely ad hoc. All claim to (more or less) fit the experimental data.

Since we have definite predictions for $V(R), V_{T}(R)$, and $V_{S}(R)$ as given above, and our confining potential, although similar, is not exactly the form used by others, it appears to be necessary to calculate the masses and the splittings of heavy-quark bound states directly. These calculations are now in progress.

\section{ACKNOWLEDGMENTS}

The work of M.B. was supported in part by the U.S. Dept. of Energy under Contract No. DOE/ER/40614. The work of J.S.B. was supported in part by the U.S. National Science Foundation Grant No. PHY 9008482. The work of F.Z. was supported in part by the U.S. Dept. of Energy under Contract No. DEAC-03-81ER40050.
[1] M. Baker, J.S. Ball, and F. Zachariasen, Phys. Rev. D 44, 3328 (1991). We shall refer to this paper as I.

[2] J.S. Ball and A. Caticha, Phys. Rev. D 37, 524 (1988).

[3] M. Baker, J.S. Ball, and F. Zachariasen, Phys. Rev. D 37, 1036 (1988).
[4] M. Baker, J.S. Ball, and F. Zachariasen, Phys. Rev. D 38, 1926 (1988).

[5] W. Lucha, F. Schöberl, and D. Gromes, Phys. Rep. 200, 129 (1991). 